\title{
Primary pure carcinoid tumour of the testis: A case report and review of the literature
}

\author{
Hideki Takada $^{1}$, Shoichiro Iwatsuki ${ }^{1}$, Yasunori Itoh ${ }^{1}$, Shinya Sato ${ }^{2,3}$, Masa Hayase ${ }^{4}$, Takahiro Yasui ${ }^{4}$ \\ ${ }^{1}$ Department of Nephro-urology, Nagoya City West Medical Center, Japan; \\ 2 Department of Experimental Pathology and Tumor Biology, Nagoya City University Graduate School of Medical Sciences, Japan; \\ ${ }^{3}$ Department of Diagnostic Pathology, Nagoya City West Medical Center, Japan; \\ ${ }^{4}$ Department of Nephro-urology, Nagoya City University Graduate School of Medical Sciences, Japan.
}

The authors contributed equally.

\begin{abstract}
Summary Primary testicular carcinoid tumours (TCT) are very rare, and a large tumour size and the presence of carcinoid syndrome predict a malignant course. Histologically, it is difficult to differentiate between benign and malignant TCTs. We report a case of a primary pure TCT with an unusual presentation in a 23year-old man, who had an asymptomatic, enlarged scrotum on the right side for 7 years. On gross examination, the tumour was $9.6 \mathrm{~cm}$ in diameter. The Ki-67 labelling index was $19.8 \%$. High inguinal orchidectomy was performed, and 30 months after surgery the patient remains asymptomatic.
\end{abstract}

KEY WORDS: Primary pure carcinoid tumour; Prognosis; Testicular cancer.

Submitted 11 May 2016; Accepted 6 June 2016

\section{INTRODUCTION}

Testicular carcinoid tumours (TCTs) are rare neoplasms, accounting for $0.2-1 \%$ of all testicular tumours $(1,2)$. To date, approximately 60 TCT cases have been described in the literature $(2,3)$. TCTs can be divided into three subgroups: primary pure TCTs, carcinoid tumours associated with teratomas ( $20 \%$ of cases), and carcinoid metastasis to the testis ( $~ 9 \%$ of cases) (4). Upon detection of a TCT, a multimodal approach should be taken to exclude the possibility of a metastasis from another organ. The mean age of patients with TCTs at diagnosis is 46 years (range, 10-83 years (4). Patients with carcinoid tumours may present with a self-detected testicular mass or testicular ache (as occurs with common testicular tumours) or, more infrequently, carcinoid syndrome with red-hot flushing (face, neck, and upper chest), severe and debilitating diarrhoea, abdominal pain, palpitations, and bronchospasms (5).

Herein, we report on a young patient with a primary pure TCT having a high malignant potential, and discuss the clinicopathological features, diagnosis, treatment, and prognosis in relation to the relevant literature.

\section{Case report}

A 23-year-old man had an asymptomatic, enlarged scrotum on the right side for 7 years. He did not have a his- tory of testicular trauma, haematuria, undescended testis, systemic symptoms, or weight loss, and there was no family history of testicular cancer. Physical examination revealed a hard, non-tender testicular mass in the right testis. The left testis appeared normal. Magnetic resonance imaging revealed a multilocular cystic tumour, measuring $8.5 \times 7.8 \times 9.6 \mathrm{~cm}$ in the right testis (Figure 1). Betahuman chorionic gonadotropin and alpha-fetoprotein lactate dehydrogenase levels were normal. Computed tomography scanning of the chest, abdomen, and pelvis revealed no significant para-aortic or iliac lymphadenopathy, no pulmonary abnormality, and no intestinal tumour. These findings favoured the diagnosis of a primary testicular tumour. The patient underwent high inguinal orchidectomy of the right testis.

Gross examination revealed that the right testis measured $9.0 \times 8.5 \times 9.6 \mathrm{~cm}$.

Cut sections of the tumour demonstrated a lobulated cystic lesion with more than one yellowish septum, and regions of haemorrhage and necrosis.

\section{Figure 1.}

Magnetic resonance imaging revealed a multilocular cystic tumour, measuring $8.5 \times 7.8 \times 9.6 \mathrm{~cm}$ in the right testis.

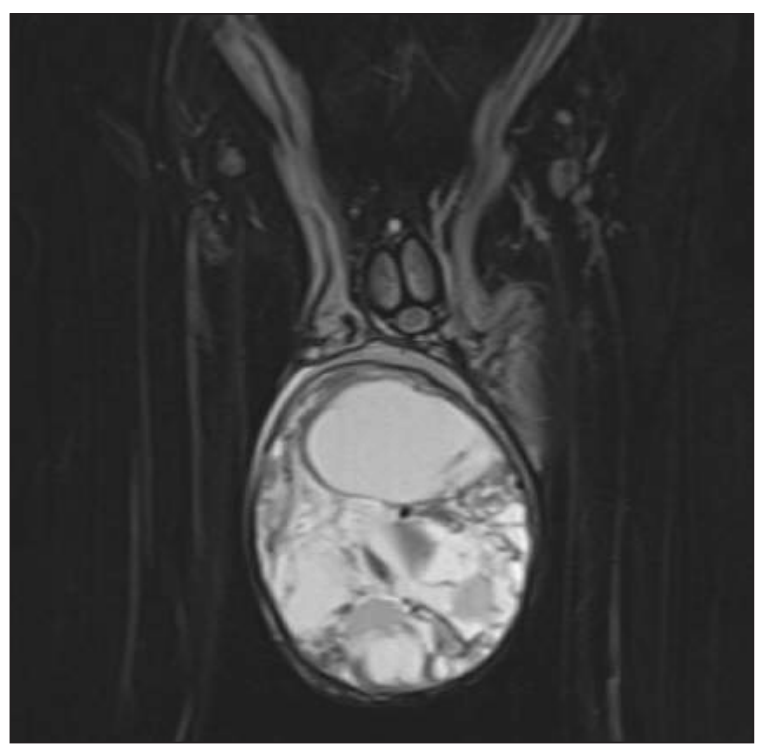


Figure 2.

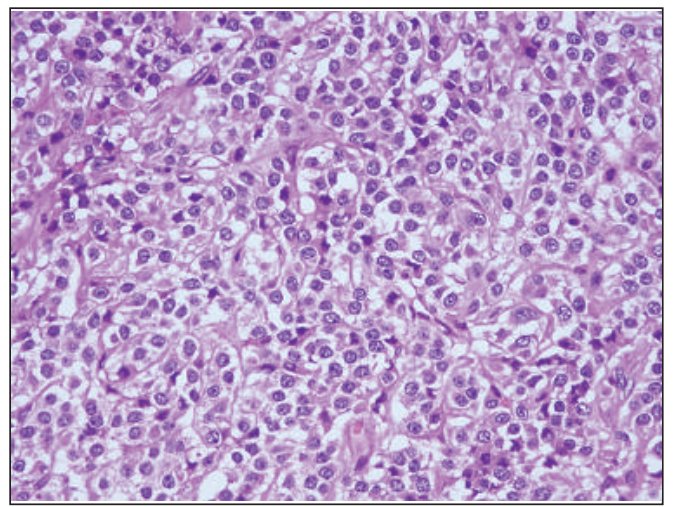

A) Microscopic appearance of the tumour showing monomorphic cells arranged in a nested trabecular pattern (haematoxylin \& eosin-stained, ×40 magnification);

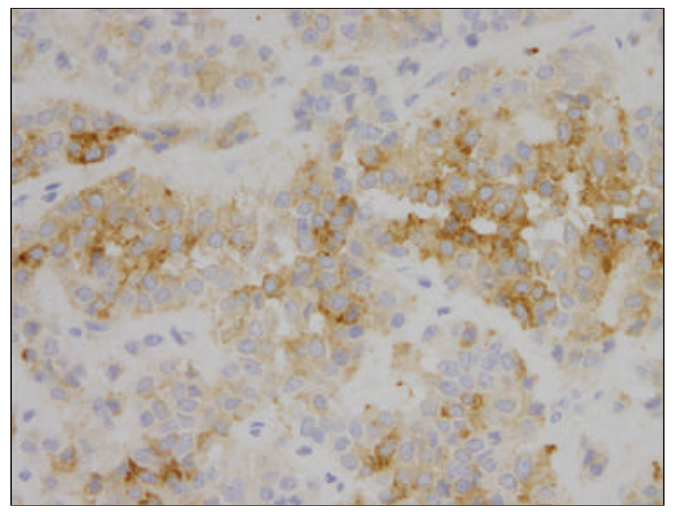

B) Tumour cells exhibiting strong immunopositivity for synaptophysin (×40 magnification).

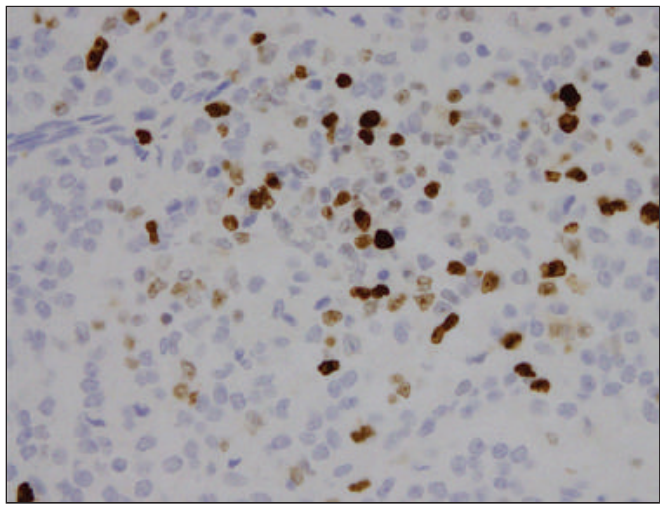

C) Ki-67 positive cells ( $\times 40$ magnification).

The tumour was confined to the testis and epididymis without lymphovascular invasion. Histology revealed monomorphic cells arranged in a nested trabecular pattern (Figure 2a). The tumour cells were circular or polygonal with round to oval nuclei, a distinct nuclear membrane, granular cytoplasm, and 'salt and pepper'-like chromatin. An immunohistochemical examination revealed the tumour cells to be strongly immunopositive for synaptophysin, facilitating the diagnosis of a neuroendocrine tumour (NET) (Figure 2b), but negative for inhibin, haematopoietic progenitor cell antigen, epithelial membrane antigen, and calretinin. The Ki-67 labelling index was $19.8 \%$ (Figure 2c).

No teratomatous elements were identified, and a diagnosis of primary pure TCT was confirmed. At 30 months after surgery, the patient remains asymptomatic.

\section{Discussion and Supplementary References are posted on www.aiua.it}

\section{Conclusion}

Our case adds to the other reports in the literature of primary TCTs having a high malignant potential. Our patient should undergo frequent biochemical and radiological examinations and long-term monitoring for recurrence and the development of metastases.

\section{References}

1. Rathert M, Ubrig B, Atkins DJ, Roth S. Carcinoid tumor of the testis. Urologe A. 2011; 50:340-2.

2. Alsharif S, Al-Shraim M, Alhadi A, et al. Primary neuroendocrine tumor of the testis. Urol Ann. 2014; 6:173-5.

3. Zavala-Pompa A, Ro JY, el-Naggar A, et al. Primary carcinoid tumor of testis. Immunohistochemical, ultrastructural, and DNA flow cytometric study of three cases with a review of the literature. Cancer. 1993; 72:1726-32.

4. Stroosma OB, Delaere KP. Carcinoid tumours of the testis. BJU Int. 2008; 101:1101 5.

5. Merino J, Zuluaga A, Gutierrez-Tejero F, et al. Pure testicular carcinoid associated with intratubular germ cell neoplasia. J Clin Pathol. 2005; 58:1331-3.

\section{Correspondence}

Hideki Takada, MD

htakada@cronos.ocn.ne.jp

Shoichiro Iwatsuki, MD

Yasunori Itoh, MD

Department of Nephro-urology, Nagoya City West Medical Center 1-1-1 Hirate-mati Kita-ku Nagoya Aichi-462-8508, Nagoya, Japan

Shinya Sato, MD

Department of Experimental Pathology and Tumor Biology,

Nagoya City University Graduate School of Medical Sciences

Department of Diagnostic Pathology, Nagoya City West Medical Center Nagoya, Japan

Masa Hayase, MD

Takahiro Yasui, MD

Department of Nephro-urology, Nagoya City University Graduate School of Medical Sciences Nagoya, Japan 